\title{
EDITORIAL
}

\section{Eosinophilic esophagitis: current status and future directions}

\author{
Pediatric Research (2020) 88:345-347; https://doi.org/10.1038/s41390- \\ 020-0770-4
}

Eosinophilic esophagitis (EoE) is a chronic, immune-mediated, inflammatory condition characterized by symptoms of esophageal dysfunction and eosinophil-predominant inflammation ( $\geq 15$ eosinophils per high-power field) of the esophageal mucosa diagnosed after exclusion of other causes of esophageal eosinophilia (Table 1). In this communication, we touch on current status of various aspects of EoE and highlight opportunities for future work.

\section{EPIDEMIOLOGY}

Until a couple of decades ago, EoE was considered an uncommon entity but has now emerged as a common cause of upper gastrointestinal $(\mathrm{Gl})$ tract morbidity in both children and adults. A large meta-analysis estimated incidence and prevalence rates in children of 5.1 cases/100,000 persons/year and 19.1 cases $/ 100,000$ persons, respectively. ${ }^{1}$ EoE is diagnosed in $2-6.5 \%$ of patients undergoing esophagogastroduodenoscopy (EGD) for any indication and this increases to $12-22 \%$ if dysphagia is the indication. ${ }^{2}$ While EoE can affect patients of all age groups, it has bimodal peak with most cases in either pediatric age group or the third decade of life. ${ }^{3}$ EoE is more common in Caucasian males and has a strong association with concomitant atopic conditions such as asthma, eczema, rhinitis, and food allergies. ${ }^{4}$

Future directions/unmet needs

The exact reasons behind the increasing incidence and prevalence are largely unknown and cannot be solely explained by increased disease recognition. There is significant interest in unraveling these changes including epidemiological factors such as early life events, infectious processes, environmental agents, and dietary habits.

\section{CLINICAL PRESENTATION}

The clinical presentation of EoE varies depending on the patient's age. Young children usually present with nonspecific symptoms such as feeding difficulties, vomiting, abdominal pain, failure to develop normal eating patterns such as not advancing past liquids/ soft solids, and occasionally failure to thrive. Adolescents and adults present with classic symptoms that include dysphagia and food impaction; EoE is now one of the most common causes of dysphagia and food impaction in the United States. ${ }^{2}$ Some patients may also present with symptoms mimicking gastro-esophageal reflux such as heartburn, epigastric pain, dyspepsia, and chest pain. Patients with EoE often develop coping mechanisms to compensate for their symptoms such as eating slowly, chewing excessively, taking small bites, cutting foods into smaller pieces, lubricating food with different sauces, drinking fluid after most bites, and avoiding textured food such as meat and bread. ${ }^{4}$

Future directions/unmet needs

Patient reported outcomes (PRO) in EoE need further study including identification and validation of instruments aside of existing Pediatric Eosinophilic Esophagitis Symptom Scores (PEESS v2.0) and PedsQL. ${ }^{5,6}$ EoE patients have chronic symptoms and may develop coping mechanisms with underappreciation of true symptoms. In the future, precision medicine may be able to specifically target different therapeutic regimens based on different clinical, endoscopic, and histologic characteristics of EoE; some of this work can be informed by the EoE endotypes recently described. ${ }^{7}$

\section{DIAGNOSIS}

The diagnosis of EoE is made based on symptoms, endoscopic features, and histological findings and EGD with biopsies is considered the diagnostic test of choice. Common endoscopic features include mucosal edema, loss of vascular pattern, linear furrows, white specks, and concentric rings. Since the esophageal mucosa may appear normal in some patients, it is imperative that esophageal mucosal biopsies be obtained in all cases as diagnosis may otherwise be missed. Due to the patchy nature of EoE, it is recommended to obtain multiple biopsies from proximal and distal esophagus. Biopsies from patients with EoE show $\geq 15$ intraepithelial eosinophils per highpower field; other findings include basal cell hyperplasia, vascular papillae elongation, eosinophil microabscesses, and lamina propria fibrosis. It is important to consider other conditions, such as gastroesophageal reflux, that can lead to esophageal eosinophilia. ${ }^{8}$

Laboratory and radiologic tests tend to be of limited utility in evaluation of patients with EoE. Contrast studies of the upper GI tract are not sensitive enough to detect subtle changes of EoE but can help identify alternate pathology or complications of EoE such as esophageal stricture and small-caliber esophagus. The role of endoluminal functional luminal imaging (EndoFLIP $\left.{ }^{\circledR}\right)$, an endoscopic tool, is being studied to assess the distensibility of the esophagus to identify early fibro-stenotic disease. ${ }^{9}$

Future directions/unmet needs

Due to invasive nature of EGD required for diagnosis and disease monitoring, there is a quest for finding noninvasive biomarkers and office-based minimally invasive tests. While several biomarkers such as eosinophil granule proteins and eosinophilic surface and intracellular markers have been investigated, the data are limited and various variables including concomitant atopy needs to be accounted for. In a proof of concept study, urine 3Bromotyrosine (3-BT) differed between EoE and atopic and nonatopic controls. ${ }^{10,11}$ Other tests being studied for diagnosis and long-term monitoring of EoE include esophageal string test and swallowed cytosponge test, which can potentially serve as minimally invasive tools to assess disease activity; initial data show good correlation with esophageal eosinophilia. Unsedated in-office transnasal endoscopy may provide another less invasive method for disease monitoring. ${ }^{12}$

\section{TREATMENT}

Treatment of EoE consists of dietary, pharmacological, and endoscopic interventions (Fig. 1). ${ }^{8}$ 


\section{Dietary therapy}

Dietary modification is an effective treatment in a significant number of patients with EoE. Three dietary methods have gained widespread acceptance: (1) empiric elimination diet: this is based on empiric elimination of most common allergens. Four-food elimination is a commonly used approach in which dairy, soy,

\begin{tabular}{|c|c|}
\hline Area & Potential opportunities \\
\hline \multirow[t]{2}{*}{ Epidemiology } & $\begin{array}{l}\text { Examine role of life factors, environment, } \\
\text { antibiotics etc. }\end{array}$ \\
\hline & Define impact of dietary habits \\
\hline Pathogenesis & $\begin{array}{l}\text { Evaluate role of cellular changes beyond } \\
\text { eosinophil }\end{array}$ \\
\hline \multirow[t]{2}{*}{ Clinical Presentation } & $\begin{array}{l}\text { Validate and refine PROs and identify } \\
\text { newer PROs }\end{array}$ \\
\hline & $\begin{array}{l}\text { Deeper understanding of EoE subtypes and } \\
\text { clinical care }\end{array}$ \\
\hline \multirow[t]{2}{*}{ Diagnosis } & Explore new biomarkers and surrogate markers \\
\hline & Study role of novel noninvasive tests \\
\hline \multirow[t]{5}{*}{ Treatment } & Refine dietary interventions \\
\hline & Compare efficacy of various steroid formulations \\
\hline & Position biologics in treatment algorithm \\
\hline & Identify role of endoscopic therapies \\
\hline & Better define therapeutic goals/endpoints \\
\hline \multirow[t]{2}{*}{ Natural history } & $\begin{array}{l}\text { Describe long-term outcomes of treated and } \\
\text { untreated disease }\end{array}$ \\
\hline & $\begin{array}{l}\text { Unravel racial, gender, age, access to care } \\
\text { disparities }\end{array}$ \\
\hline
\end{tabular}

wheat, and eggs are empirically eliminated. Newer approaches with "step up" empiric diet are also being studied; (2) elemental diet: in this regimen all foods are replaced with an elemental, amino acid-based formula; (3) targeted elimination diet: this involves the elimination of foods based on allergy testing, though diagnostic accuracy can be a problem owing to false-positive and false-negative test results.

Compliance and nutritional deficiencies associated with these dietary approaches can be a challenge and a close follow-up with an experienced dietician is important.

\section{Pharmacologic therapy}

Proton-pump inhibitors (PPI) are an important pharmacologic treatment option for EoE. In addition to acid suppression properties, PPIs have anti-inflammatory properties that impact pathobiology of EoE. Until recently, an 8-week trial of high-dose PPI with follow-up EGD was recommended for patients with esophageal eosinophilia and responders were referred to as PPI-responsive esophageal eosinophilia (PPI-REE). Recently revised guidelines no longer mandate such trial prior to establishing EoE diagnosis but rather position PPI as a treatment modality for EoE. ${ }^{13}$

Topical glucocorticoids (TCS) including budesonide and fluticasone are other pharmacological options and both are effective at improving symptoms and histology in patients with EoE. A variety of administration methods have been used to deliver these drugs including as viscous slurry, metered-dose inhaler, and effervescent tablet. Possible adverse effects of topical corticosteroid preparations include local candidal overgrowth, adrenal axis suppression and suppression of height velocity. None of the pharmacological therapies are approved by the Food and Drug Administration (FDA); European Medicines Agency (EMA) has approved an oral budesonide preparation, Jorveza ${ }^{\circledR}$, for adults with EoE.

\section{Endoscopic therapy}

Role of endoscopy in the treatment of EoE is generally limited to management of complications such as food impaction removal or

\section{Algorithm}

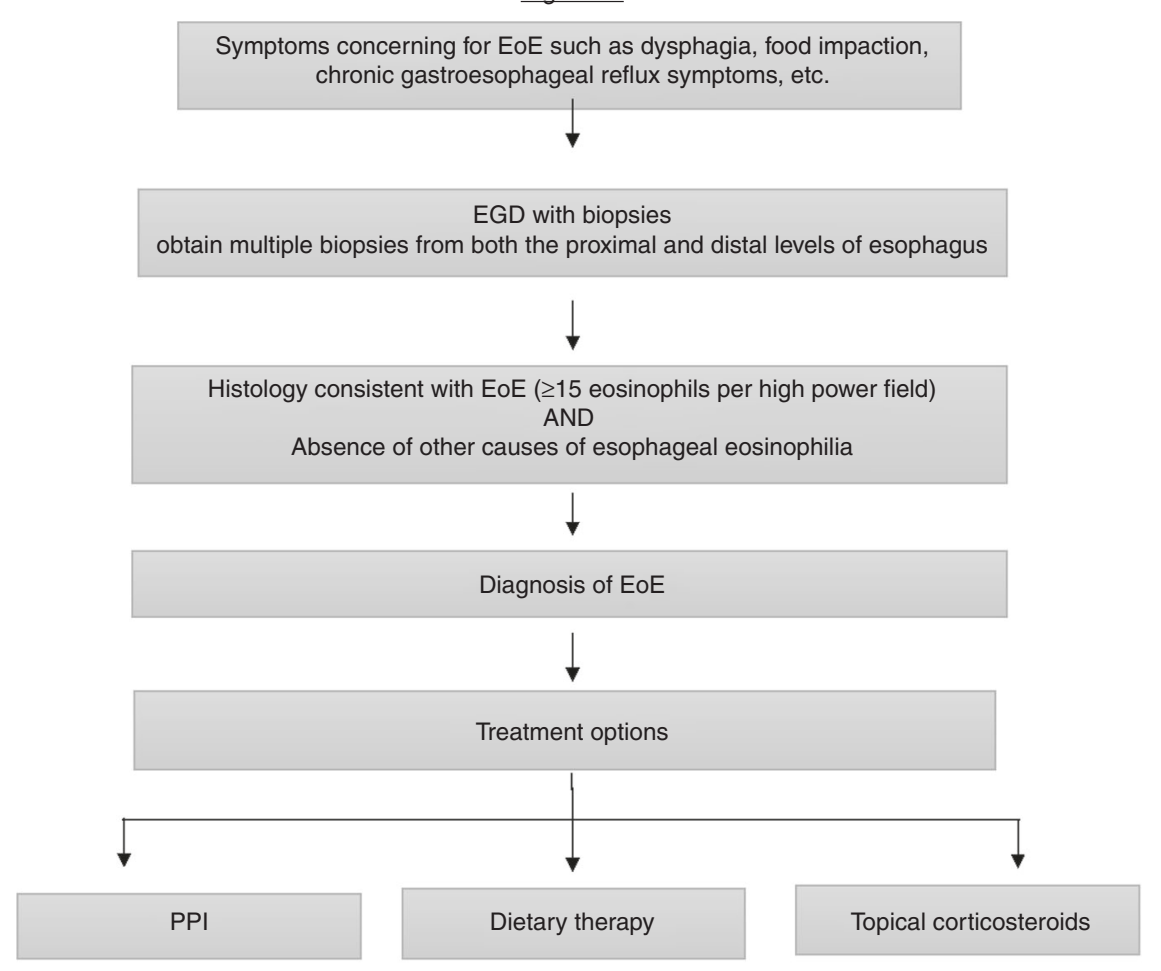

Fig. 1 Management of eosinophilic esophagitis. 
dilation of esophageal strictures that are unresponsive to medical therapy. Relapse is relatively common after dilation, especially if dilation was used as the sole modality for treatment. A study in adults of esophageal dilation outcomes reported that $58 \%$ of the patients required a second dilation, with majority (75\%) requiring within 1 year. ${ }^{14}$

Future directions/unmet needs

Multiple topical corticosteroid preparations, including viscous budesonide suspension and dissolving fluticasone tablets, are being studied in EoE to help with easier administration of medication. Several biologic drugs targeting interleukin (IL)-5 (mepolizumab and reslizumab), IL-13 (lebrikizumab), IL-4 and IL-13 (dupilumab) are being investigated as potential treatment for EoE. ${ }^{15}$ Omalizumab, an IgE monoclonal antibody, did not improve endoscopic or histological features in EoE patients. ${ }^{16}$ Further research may also assess their role in the treatment of refractory EoE.

\section{MAINTENANCE THERAPY, NATURAL HISTORY AND LONG- TERM COMPLICATIONS}

There is no clear consensus regarding appropriate maintenance regimen for EoE, a chronic condition that frequently recurs in patient who discontinue treatment. It is generally necessary to continue maintenance treatment, whether avoidance of dietary triggers or pharmacological, to maintain remission.

Knowledge on the natural history of EoE is limited and many aspects including risk-stratification for complications are incompletely defined. There, however, is data to support that chronic, unresolved inflammation results in structural damage to esophagus, leading to fibrosis, strictures, and impaired esophageal function over time. This underscores the importance of early diagnosis and need for maintenance therapy.

Future directions/unmet needs

EoE is an entity that was largely unknown until about 2-3 decades ago; hence, our understanding of various aspects of its natural history is still in its infancy. More prospective, long-term outcomebased research studies are required to understand these aspects. A better understanding of natural history, pathogenesis, and impact of current treatments on disease progression will help in shaping future clinical practice.

\section{ACKNOWLEDGEMENTS}

S.K.G. is supported in part by CEGIR (U54 Al117804) that is part of the Rare Disease Clinical Research Network (RDCRN), an initiative of the Office of Rare Diseases Research (ORDR), NCATS, and is funded through collaboration between NIAID, NIDDK and NCATS. CEGIR is also supported by patient advocacy groups including American Partnership for Eosinophilic Disorders (APFED), Campaign Urging Research for Eosinophilic Diseases (CURED), and Eosinophilic Family Coalition (EFC).

\section{AUTHOR CONTRIBUTIONS}

Each author has met the Pediatric Research authorship requirements.

\section{ADDITIONAL INFORMATION}

Competing interests: S.K.G. serves as consultant for Adare Pharmaceuticals Inc, Allakos, Abbott Laboratories, Gossamer Bio, QOL Medical LLC and Receptos, Inc. He also receives research support from Shire Pharmaceuticals.

Publisher's note Springer Nature remains neutral with regard to jurisdictional claims in published maps and institutional affiliations.

Sanjay Kumar ${ }^{1}$, Sukgi S. Choi ${ }^{2}$ and Sandeep K. Gupta ${ }^{3}$ ${ }^{1}$ Ascension St John Providence Children's Hospital, Detroit, MI, USA; ${ }^{2}$ Department of Otolaryngology and Communication Enhancement, Harvard Medical School, Boston, MA, USA and ${ }^{3}$ Section of Pediatric Gastroenterology, Hepatology and Nutrition, University of Illinois College of Medicine at Peoria/Children's Hospital of Illinois, 530 NE Glen Oak Avenue North Building, Room 6646, Peoria, IL, USA Correspondence: Sandeep K. Gupta (skgupta@uic.edu)

\section{REFERENCES}

1. Arias, A. et al. Systematic review with meta-analysis: the incidence and prevalence of eosinophilic oesophagitis in children and adults in population-based studies. Aliment Pharm. Ther. 43, 3-15 (2016).

2. Dellon, E. S. \& Hirano, I. Epidemiology and natural history of eosinophilic esophagitis. Gastroenterology 154, 319-332.e3 (2018).

3. Benninger, M. S. et al. Prevalence of atopic disease in patients with eosinophilic esophagitis. Int. Forum Allergy Rhinol. 7, 757-762 (2017).

4. Kumar, S., Choi, S. \& Gupta, S. K. Eosinophilic esophagitis-a primer for otolaryngologists. JAMA Otolaryngol. Head Neck Surg. 145, 373-380 (2019).

5. Martin, L. J. et al. Pediatric Eosinophilic Esophagitis Symptom Scores (PEESS v2.0) identify histologic and molecular correlates of the key clinical features of disease. J. Allergy Clin. Immunol. 135, 1519-28.e8 (2015).

6. Franciosi, J. P. et al. PedsQL eosinophilic esophagitis module: feasibility, reliability, and validity. J. Pediatr. Gastroenterol. Nutr. 57, 57-66 (2013).

7. Shoda, T. et al. Eosinophilic oesophagitis endotype classification by molecular, clinical, and histopathological analyses: a cross-sectional study. Lancet Gastroenterol. Hepatol. 3, 477-488 (2018).

8. Dellon, E. S. et al. ACG clinical guideline: evidenced based approach to the diagnosis and management of esophageal eosinophilia and eosinophilic esophagitis (EoE). Am. J. Gastroenterol. 108, 679-692 (2013). quiz 693.

9. Menard-Katcher, C. et al. Influence of age and eosinophilic esophagitis on esophageal distensibility in a pediatric cohort. Am. J. Gastroenterol. 112, 1466-1473 (2017).

10. Cunnion, K. M. et al. Eosinophil quantitated urine kinetic: a novel assay for assessment of eosinophilic esophagitis. Ann. Allergy Asthma Immunol. 116 435-439 (2016).

11. Hines, B. T. et al. Minimally invasive biomarker studies in eosinophilic esophagitis: a systematic review. Ann. Allergy Asthma Immunol. 121, 218-228 (2018).

12. Hiremath, G. \& Gupta, S. K. Promising modalities to identify and monitor eosinophilic esophagitis. Clin. Gastroenterol. Hepatol. 15, 1655-1664 (2017).

13. Dellon, E. S. et al. Updated International Consensus Diagnostic Criteria for Eosinophilic Esophagitis: Proceedings of the AGREE Conference. Gastroenterology 155, 1022-1033.e10 (2018).

14. Runge, T. M. et al. Outcomes of esophageal dilation in eosinophilic esophagitis: safety, efficacy, and persistence of the fibrostenotic phenotype. Am. J. Gastroenterol. 111, 206-213 (2016).

15. Pesek, R. D. \& Gupta, S. K. Emerging drugs for eosinophilic esophagitis. Expert Opin. Emerg. Drugs 23, 173-183 (2018).

16. Rocha, R. et al. Omalizumab in the treatment of eosinophilic esophagitis and food allergy. Eur. J. Pediatr. 170, 1471-1474 (2011). 